\title{
Comparison of Expression Vectors for Transient Expression of Recombinant Proteins in Plants
}

\author{
Kausar Hussain Shah • Bachar Almaghrabi • \\ Holger Bohlmann \\ Published online: 25 July 2013 \\ (C) The Author(s) 2013. This article is published with open access at Springerlink.com
}

\begin{abstract}
Production of recombinant proteins in plants is of increasing importance for practical applications. However, the production of stable transformed transgenic plants is a lengthy procedure. Transient expression, on the other hand, can deliver recombinant proteins within a week, and many viral vectors have been constructed for that purpose. Each of them is reported to be highly efficient, robust and costeffective. Here, a variety of expression vectors which were designed for transient and stable plant transformation, including pPZP3425, pPZP5025, pPZPTRBO, pJLTRBO, pEAQ- $H T$ and $\mathrm{pBY} 030-2 \mathrm{R}$, was compared for the expression of green fluorescent protein and $\beta$-glucuronidase in Nicotiana benthamiana by Agrobacterium-mediated transient expression. Our results show that pPZPTRBO, pJLTRBO and pEAQ- $H T$ had comparable expression levels without co-infiltration of a RNA-silencing inhibitor. The other vectors, including the non-viral vectors pPZP5025 and pPZP3425, needed co-infiltration of the RNA-silencing inhibitor P19 to give good expression levels.
\end{abstract}

Keywords Transient expression - Recombinant protein . Agroinfiltration $\cdot$ Viral vector $\cdot \mathrm{pPZP}$ vector family

\section{Abbreviations \\ CaMV Cauliflower mosaic virus \\ dpi Days post infiltration}

Electronic supplementary material The online version of this article (doi:10.1007/s11105-013-0614-z) contains supplementary material, which is available to authorized users.

K. H. Shah • B. Almaghrabi • H. Bohlmann $(\triangle)$ Division of Plant Protection, Department of Crop Sciences, UFT, University of Natural Resources and Life Sciences, Vienna, Konrad Lorenz Strasse 24, 3430 Tulln, Austria

e-mail: holger.bohlmann@boku.ac.at

K. H. Shah

e-mail: kausar.shah@boku.ac.at

B. Almaghrabi

e-mail: bachar.almaghrabi@boku.ac.at $\begin{array}{ll}\text { HR } & \text { Hypersensitive response } \\ \text { 4-MU } & \text { 4-Methylumbelliferone } \\ \text { 4-MUG } & \text { 4-Methylumbelliferyl- } \beta \text {-D-glucuronide } \\ \text { TBSV } & \text { Tomato bushy stunt virus } \\ \text { TMV } & \text { Tobacco mosaic virus } \\ \text { TSP } & \text { Total soluble protein }\end{array}$

\section{Introduction}

The production of recombinant proteins is of major interest for basic and applied research. There are several well-established systems in use for this purpose, e.g. Escherichia coli, yeast, mammalian cells and plant cells. Among these systems, plant cell factories have many advantages. Large quantities of plants can be grown easily at low cost, and when compared to mammalian cell cultures, there is no risk of contamination with retroviruses. Plant cells have an endomembrane system and secretory pathway similar to mammalian cells which allows post-translational modification of recombinant proteins (Pogue et al. 2010; Popescu et al. 2007). However, plants have the limitation that it usually takes a long time to produce stable transformed transgenic plants as compared to other expression systems, but this problem can be solved by transient expression methods. Transient expression in plant leaves enables the small- to medium-scale production of recombinant proteins at a timescale that is comparable to other prokaryotic and eukaryotic expression systems. The preferred host plant for transient expression is Nicotiana benthamiana (Conley et al. 2011; Goodin et al. 2008; Sheludko et al. 2007). It is very susceptible to plant viruses, particularly due to a defective form of a RNA-dependent RNA polymerase found in its genome (Yang et al. 2004). This viral susceptibility allows external viral replicases to replicate the delivered genes. The combination of ease of infection with bacterial and viral components and a long history of experimental use have made $N$. benthamiana a common laboratory host for the expression 
of recombinant proteins. This expression platform is based on leaves, and it significantly reduces the potential for gene leakage into the environment through pollen or seed dispersal. Furthermore, N. benthamiana is a non-food, non-feed crop, which minimizes the risk of recombinant proteins entering the food chain (Rymerson et al. 2002; Twyman et al. 2003). It has, for instance, been used to produce the haemagglutinin antigen from avian influenza virus A (Kanagarajan et al. 2012) and for the production of monoclonal antibodies (Castilho et al. 2011). Furthermore, it should be mentioned that transient expression, especially in $N$. benthamiana, is also intensively used to analyse the function of proteins (for instance, Hashimoto et al. 2012).

Transient expression of foreign proteins in plants has been mediated via simple tissue infiltration using Agrobacterium tumefaciens (Kapila et al. 1997) or by vectors based on the genomes of a variety of plant viruses. Furthermore, viral vectors have also been integrated into binary vectors, such that the replicating vector is delivered and released into the host tissues by infiltration with recombinant agrobacteria (Gleba et al. 2005; Van der Hoorn et al. 2000; Vaquero et al. 1999). The simple integrative plant expression vector approach has the disadvantage that expression levels are usually low and decline after a few days. However, ectopic expression of RNA-silencing suppressor proteins such as P19 from tomato bushy stunt virus or HC-Pro from tobacco etch virus results in significantly increased expression of the desired proteins (Voinnet et al. 2003). The virus-based vectors offer advantages over simple T-DNA vectors by exploiting the cytoplasmic replication cycle of the virus. A large number of viral vectors have been designed which can be classified as gene substitution vectors, gene insertion vectors, modular or deconstructed vector systems and peptide display vectors (Lico et al. 2008). The first generation virus vectors were gene substitution vectors or gene insertion vectors that were inoculated as virus particles or viral RNA and used virus-encoded cell-to-cell and systemic movement activities to infect host plants (Gleba et al. 2007; Scholthof 2004). In substitution vectors, a viral gene, usually the coat protein gene, is replaced by a gene encoding the protein to be expressed. The loss of the coat protein usually limits the cellto-cell and systemic movement in the plant, thus leading to low expression levels if the inoculation depends on virus particles or viral RNA. This problem can be solved if the viral vector is introduced by agroinfiltration into the majority of plant cells, thereby bypassing the need for cell-to-cell and systemic movement. The gene insertion vectors are complete (functional) viruses with an additional coding sequence which is transcribed from a viral promoter (Lico et al. 2008). Since the packaging capacity of most virus envelops is close to the size of the wild-type genome, these vectors are not useful for the production of large proteins. In modular vector systems, different parts of the virus vector are placed on separate binary vectors. As these viral vectors do not show systemic movement in inoculated plants, agroinfiltration is used to transfer the viral constructs to the tissues of host plants to launch the infection process. The best known example of these vectors has been developed by the company Icon Genetics (Halle, Germany). The system has, for instance, been used to produce different antibodies (Giritch et al. 2006; Gils et al. 2005). It is reported that it gives expression levels of up to $5 \mathrm{~g}$ recombinant proteins per $\mathrm{kg}$ fresh weight of infiltrated tissues (Gleba et al. 2005). In deconstructed vector systems, some unnecessary parts of the viral genome are removed such as deletion of coat protein gene. The vector pEAQ- $H T$ was reported as an efficient transient expression vector which is based on a deleted version of the Cowpea mosaic virus that permits high-level and rapid production of proteins $(0.2-0.5 \mathrm{~g}$ per kg fresh weight (FW) of infiltrated tissues) without viral replication (Sainsbury et al. 2008, 2009). The vector pBY030$2 \mathrm{R}$ is a bean yellow dwarf virus single-stranded DNA-based vector (Huang et al. 2009; Mor et al. 2003) which harbours a long intergenic region (LIR), a short intergenic region (SIR) and two open reading frames ( $\mathrm{C} 1$ and $\mathrm{C} 2$ ), encoding replication-associated proteins Rep and RepA, respectively, within the T-DNA (Liu et al. 1997). This vector was reported to produce $0.5 \mathrm{mg}$ of recombinant proteins per $\mathrm{g} \mathrm{FW}$ of the infiltrated tissues (Huang et al. 2009).

Another example of the degenerated viral vectors is the pJLTRBO (tobacco mosaic virus (TMV) RNA-based overexpression) vector which is an improved agroinfectioncompatible TMV vector that lacks the TMV coat protein gene and is therefore unable to form virus particles during the infection process (Lindbo 2007). It was reported to have high agroinfection efficiency and a high recombinant protein expression level of 3-5 $\mathrm{mg}$ of recombinant proteins per $\mathrm{g} \mathrm{FW}$ of infiltrated tissues.

In this study, a variety of viral and binary plant transformation vectors were compared for their ability to produce recombinant proteins in transient expression in $N$. benthamiana. In addition to the above-mentioned viral vectors, the binary vectors pPZP3425 (Szakasits et al. 2007) and pPZP5025 (Ali et al. 2012) were included.

\section{Materials and Methods}

\section{Vector Construction}

The vectors pPZP3425, pJLTRBO, pEAQ-HT-GFP and pBY030-2R were previously reported (Szakasits et al. 2007; Lindbo 2007; Sainsbury et al. 2009; Huang et al. 2009). The T-DNA region of all the vectors used in this study is shown in Fig. 1. The vectors pPZP5025 and pPZPTRBO are based on the modified high-copy-number pPZP family (Hajdukiewicz et al. 1994) vector pPZP500 


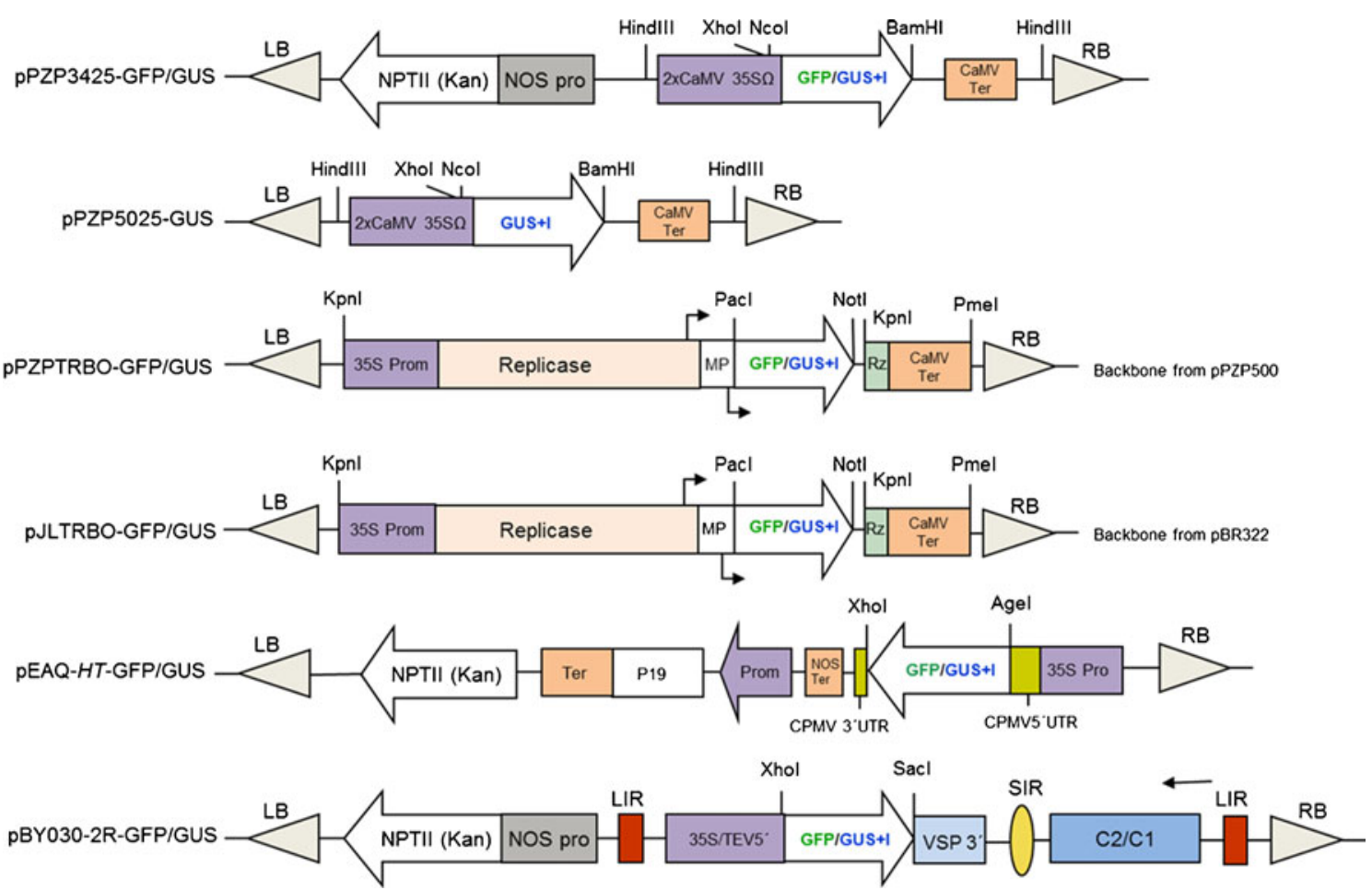

Fig. 1 T-DNA regions of the vectors used in this study. NOS, nopaline synthase promoter; $\mathrm{Ter}$, CaMV transcription terminator; $R z$, sequence for ribozyme; $M P$, CaMV movement protein sequence; $5^{\prime}-U T R$ and $3{ }^{\prime}-$ UTR, sequences from cowpea mosaic virus RNA-2; NPTII, neomycin phosphotransferase (kanamycin resistance); $T E V$, tobacco etch virus; $S I R$ and LIR, short and long intergenic regions of BeYDV genome; vsp3', soybean vspB gene $3^{\prime}$ region; $C 1 / C 2$, BeYDV ORFs encoding replication initiation protein (Rep) and RepA; bent arrows, subgenomic promoters; $I$, intron; $\Omega$, omega translational enhancer element from TMV; $P 19,19 \mathrm{kDa}$ RNA-silencing suppressor gene from tomato bushy stunt virus; $L B$ and $R B$, left and right borders of the T-DNA region
(Ali et al. 2012). The vector pPZP5025 was produced by inserting the $\beta$-glucuronidase (GUS) cassette from pPZP3425 as a HindIII fragment into the same site of pPZP500. The vector pPZPTRBO contains the TMV cassette from pJLTRBO and was constructed as follows: the cauliflower mosaic virus (CaMV) terminator plus the ribozyme sequence from pJLTRBO was cloned as a KpnI/PmeI fragment in pPZP500 digested with the same enzymes to produce pPZP500-Ter. The sequence of the terminator region was confirmed by sequencing using the primer P500Ter forward (for) (5'-GCTGGTGGCAGGATATATTG-3'). The TMV cassette containing the enhanced $35 \mathrm{~S}$ promoter, replicase gene, movement protein gene and polylinker was cut out from pJLTRBO as a KpnI fragment and ligated to the same site of pPZP500-Ter to generate pPZPTRBO (Figs. 1 and $\mathrm{S} 1$ ).

The GFP coding sequence was cut out as a NcoI-BamHI fragment from an intermediate cloning vector (pUC-HB3GFP) and used to replace the GUS coding sequence in pPZP3425 to produce pPZP3425-GFP, and it was inserted as a NotI-PacI fragment into pPZPTRBO and pJLTRBO to produce $\mathrm{pPZPTRBO-GFP}$ and pJLTRBO-GFP, respectively (Fig. 1). The intron containing GUS sequence from pPZP3425 was cloned as a NcoI-BamHI fragment into pUC-HB3 (Bohlmann, unpublished) from which it was cut out as a NotI-PacI fragment and cloned into pPZPTRBO and pJLTRBO to produce pPZPTRBO-GUS and pJLTRBO-GUS, respectively. The vectors pBY030-2R-GUS and pEAQ-HTGUS were produced as follows: the GUS fragment was amplified from pUCHB3-GUS with the oligos P-XhoI for (5'CAGCTCGAGCATGGTCCGTCCTGTAGAAACC-3') and P-SacI rev (5'-AGTGAGCTCCCCACCGAGGCTGTAG-3'), digested with XhoI and SacI and cloned into pBY030-2R digested with XhoI and SacI. Similarly, the GUS fragment was also amplified from pUC-HB3-GUS with the oligos PAgeI for (5'-CTTAACCGGTCCATGGTCCGTCCT-3') and PXhoI rev (5'-GACTCTCGAGCGGCCGCGGAT-3'), digested with AgeI and XhoI and cloned into pEAQ- $H T$ digested with AgeI and XhoI. The vector pBin61-P19 (Voinnet et al. 2003) was used to express the P19 RNA-silencing suppressor.

Agroinfiltration

$N$. benthamiana plants were grown in a growth chamber at $25 \pm 2{ }^{\circ} \mathrm{C}$ temperature, 16 -h photoperiod and $\sim 65 \%$ humidity. Binary plasmids were transformed into agrobacteria by 
the freeze-thaw method (Holsters et al. 1978). In most experiments, the strain GV3101 (Koncz and Schell 1986) was used. In addition, some experiments were performed with LBA4404 (Hoekema et al. 1983). Agrobacteria were grown overnight in a YEB liquid medium with appropriate antibiotics $(35 \mu \mathrm{g} / \mathrm{ml}$ rifampicin for both agrobacteria and $25 \mu \mathrm{g} / \mathrm{ml}$ gentamicin for GV3101, $200 \mu \mathrm{g} / \mathrm{ml}$ spectinomycin for pPZP5025 and pPZPTRBO and $50 \mu \mathrm{g} / \mathrm{ml}$ kanamycin for the rest of the plasmids) to an optical density (OD) ${ }_{600}$ of 0.8 in an incubator/shaker at $28{ }^{\circ} \mathrm{C}$. Bacteria were harvested by centrifugation at 5,000 rpm for $6 \mathrm{~min}$ in a table top centrifuge at room temperature and suspended in an infiltration medium (10 mM MES, pH 5.6; $10 \mathrm{mM} \mathrm{MgCl}_{2}$ and $100 \mu \mathrm{M}$ acetosyringone) to obtain bacterial suspensions of the required $\mathrm{OD}_{600}(0.4$ and 1.0$)$. After incubation for $2 \mathrm{~h}$, agrobacteria were infiltrated into the abaxial side of leaves by using a 1-ml syringe without needle. Infiltrated plants were kept under the same growth conditions as mentioned above. For co-infiltration of RNA-silencing inhibitor P19, an equal volume of a bacterial suspension harbouring pBin61P19 (Voinnet et al. 2003) was added prior to infiltration.

In one experiment, two leaves (leaf numbers 3 and 4 ) were infiltrated. Leaf samples were taken after 6 days unless otherwise indicated. One sample consisted of 20-30 leaf disks of $10 \mathrm{~mm}$ diameter that were cut out from 1 leaf and immediately frozen in liquid nitrogen for later analysis. All samples were analysed in triplicate. Three independent experiments were performed.

\section{GUS Staining and GFP Imaging}

GUS staining of $N$. benthamiana leaves was done by overnight incubation in X-Gluc solution $(50 \mathrm{mM}$ sodium phosphate buffer, $\mathrm{pH} 7.0 ; 10 \mathrm{mM}$ ethylenediaminetetraacetic acid (EDTA), $\mathrm{pH} 8.0 ; 0.1 \%(v / v)$ Triton X-100 and $0.5 \mathrm{mg} / \mathrm{ml} \mathrm{X-}$ Gluc) at $37{ }^{\circ} \mathrm{C}$ (Jefferson et al. 1987) followed by several washes with $70 \%$ ethanol to clear the chlorophyll from leaf tissues. The GFP fluorescence imaging was done by illuminating the infiltrated leaves under UV light with a handheld UV lamp. Images were taken with the Nikon digital camera.

\section{Protein Extraction}

Total soluble proteins (TSP) were extracted from 200 to $400 \mathrm{mg}$ FW of the infiltrated leaf tissues. Leaf disks (2030 ) were frozen in the Eppendorf tubes in liquid nitrogen, ground in a mixer mill (Retsch MM 400) and immediately dissolved in $1 \mathrm{ml}$ of extraction buffer consisting of $50 \mathrm{mM}$ Tris-HCl, pH 7.25; 150 mM NaCl; 2 mM EDTA; $0.1 \%(v / v)$ Triton X-100 for GFP quantification (Sainsbury 2009) and $50 \mathrm{mM} \mathrm{NaH}{ }_{2} \mathrm{PO}_{4}, \mathrm{pH} 7.0 ; 10 \mathrm{mM}$ EDTA; $0.1 \%(v / v)$ Triton $\mathrm{X}-100 ; 0.1 \%$ sodium lauryl sarcosine and $10 \mathrm{mM} \beta$ mercaptoethanol for GUS quantification (Jefferson et al.
1987). The extracts were centrifuged $\left(13,000 \mathrm{rpm}\right.$ at $\left.4{ }^{\circ} \mathrm{C}\right)$ for $10 \mathrm{~min}$. The supernatant was then centrifuged again for $10 \mathrm{~min}$ to clarify it from leaf tissues debris. The 1:10 dilutions of the extracts were made for quantification.

\section{Protein Quantification}

Proteins in the extracts were quantified using the Pierce ${ }^{\circledR}$ BCA Protein Assay Kit. Twenty-five microlitres of a 1:10 dilution of the extract and $200 \mu$ of the working reagent were added to a transparent 96-well microtiter plate (Greiner). After incubation for $30 \mathrm{~min}, \mathrm{OD}_{600}$ was measured at $562 \mathrm{~nm}$ with the FLUOstar Omega micro-plate reader (BMG Labtech). A standard curve was produced by using BSA standards in the range of $25-2,000 \mu \mathrm{g} / \mathrm{ml}$.

Sodium Dodecyl Sulphate-Polyacrylamide Gel Electrophoresis

TSP samples were resolved on tricine-sodium dodecyl sulphate (SDS) polyacrylamide gels (16\% separating and $4 \%$ stacking gel) according to Schägger (2006).

\section{GFP Fluorescence Measurement}

GFP relative fluorescence produced by different vectors was measured at $400 \mathrm{~nm}$ excitation and $510 \mathrm{~nm}$ emission with the FLUOstar Omega micro-plate reader (BMG Labtech) using $100 \mu \mathrm{l}$ of a 1:10 dilution of extracts (prepared as described in 'Protein Extraction') in black 96-well microtiter plates (Greiner).

\section{GUS Activity Assay}

The GUS activity was measured according to Jefferson et al. (1987). The $3 \mathrm{mM}$ solution $(50 \mu \mathrm{l})$ of the GUS substrate 4methylumbelliferyl- $\beta$-D-glucuronide (4-MUG) was added to $100 \mu \mathrm{l}$ of protein extract (prepared as described in 'Protein Extraction') in a black 96-well microtiter plate (Greiner) to obtain a final concentration of $1 \mathrm{mM} 4-\mathrm{MUG}$ in $150 \mu \mathrm{l}$ of reaction volume. The reaction was carried out by incubation at $37^{\circ} \mathrm{C}$ for $5 \mathrm{~min}$, and then, $50 \mu \mathrm{l}$ of $0.5 \mathrm{M} \mathrm{Na}_{2} \mathrm{CO}_{3}$ was used to stop the reaction. Fluorescence was measured at $355 \mathrm{~nm}$ excitation and $460 \mathrm{~nm}$ emission in the FLUOstar Omega micro-plate reader (BMG Labtech) using 4-MU standards $(10 \mathrm{mM}$ stock in ethanol and diluted in GUS extraction buffer) in the range of $1-100 \mu \mathrm{M}$.

\section{Results}

The goal of this work was to compare the efficiency of different viral and binary vectors for transient expression in 
$N$. benthamiana leaves using the GUS and GFP reporters. They have a different size (GUS has a monomeric molecular weight of $68 \mathrm{kDa}$, while that of GFP is of $27 \mathrm{kDa}$ ), and both can be measured and quantified using either the enzymatic activity of GUS or the fluorescence of GFP as described in experimental procedures. The vectors included the viral vectors pPZPTRBO (this study), pJLTRBO (Lindbo 2007), pEAQ-HT (Sainsbury et al. 2009) and pBY030-2R (Huang et al. 2009) and the binary vectors pPZP3425 (Szakasits et al. 2007) and pPZP5025 (Ali et al. 2012) (Fig. 1). The vector pJLTRBO was reported to be a high efficiency vector; however, it has a low copy number. To remove this limitation, the TMV cassette was transferred from its T-DNA to our previously designed high-copy-number vector pPZP500, thus generating pPZPTRBO (Figs. 1 and S1). The vector pPZP3425 contains a kanamycin-selectable marker and the GUS coding sequence (interrupted by a single intron) driven by a doubled CaMV $35 \mathrm{~S}$ promoter with a TMV omega translational enhancer. The vector pPZP5025 is a streamlined version of pPZP200 (Hajdukiewicz et al. 1994) without a plant-selectable marker, but containing the CaMV expression cassette from pPZP3425.

All the vectors were transformed into the disarmed $A$. tumefaciens strain GV3101. Agrobacteria were infiltrated at an $\mathrm{OD}_{600}$ of 0.4 or 1.0 into $N$. benthamiana leaves alone or together with the vector pBIN61 expressing the RNAsilencing suppressor P19 (Voinnet et al. 2003). In the paper reporting pEAQ-HT (Sainsbury et al. 2009), the authors used the Agrobacterium strain LBA4404. It was therefore tested if LBA4404 might give stronger expression levels than GV3101. The GFP fluorescence and GUS staining results for both GV3101- and LBA4404-infilrated leaves were similar (see Figs. 2 and 4 for comparison). Quantification of GFP expression by pEAQ-HT showed stronger fluorescence using GV3101 than LBA4404 (Fig. S3). In addition, expression with GV3101 gave no difference with or without P19, while infiltration in LBA4404 needed co-infiltration of P19 for best results with pEAQ-HT. Thus, GV3101 was the Agrobacterium strain of choice and was used for all other experiments.

In a first set of experiments, the vectors containing the GFP coding sequence were compared (Fig. 2). Both TMV vectors and $\mathrm{pEAQ}-H T$ resulted in a strong GFP fluorescence with and without P19, while expression in pBY030-2R was much lower and only visible with P19 co-infiltration. In the case of pBY030-2R, a hypersensitive response (HR) in the infiltrated leaves became evident after 7 days (Fig. 2). Similarly, GFP expressed by pPZP3425 was only visible after co-infiltration with $\mathrm{P} 19$, and the fluorescence level was between that of pBY030-2R and the other vectors. GFP expression was analysed at different time points after infiltration, and expression was generally higher at 5 days post infiltration (dpi) (Fig. S4). Therefore, GFP fluorescence was quantified at 5 dpi (Fig. 3). Both TMV vectors and pEAQ-HT showed the highest GFP level, and coinfiltration of P19 had a small but significant positive effect only in the case of pJLTRBO, while there was no difference for $\mathrm{pPZPTRBO}$ and $\mathrm{pEAQ}-H T$. The vector $\mathrm{pBY} 030-2 \mathrm{R}$ had the lowest GFP fluorescence level of all vectors that were compared. The GFP level without P19 was approximately seven times lower as compared to the strong expressing virus vectors but was enhanced about threefold by co-infiltration of P19. The binary vector pPZP3425 had the lowest expression level of all when used without P19 but showed the
Fig. 2 GFP fluorescence and HR induced by $\mathrm{pBY} 030-2 \mathrm{R}$ in $N$. benthamiana leaves. The $N$. benthamiana leaves were infiltrated with agrobacteria $\left(\mathrm{OD}_{600} 1.0\right)$ containing the GFP expression vectors. The GFP fluorescence was monitored under UV light at $5 \mathrm{dpi}$. The area marked with ' $+P 19$ ' indicates co-infiltration of P19. The picture in the lower right corner shows HR in the leaves after 7 days of $\mathrm{pBY} 030-2 \mathrm{R}$ infiltration
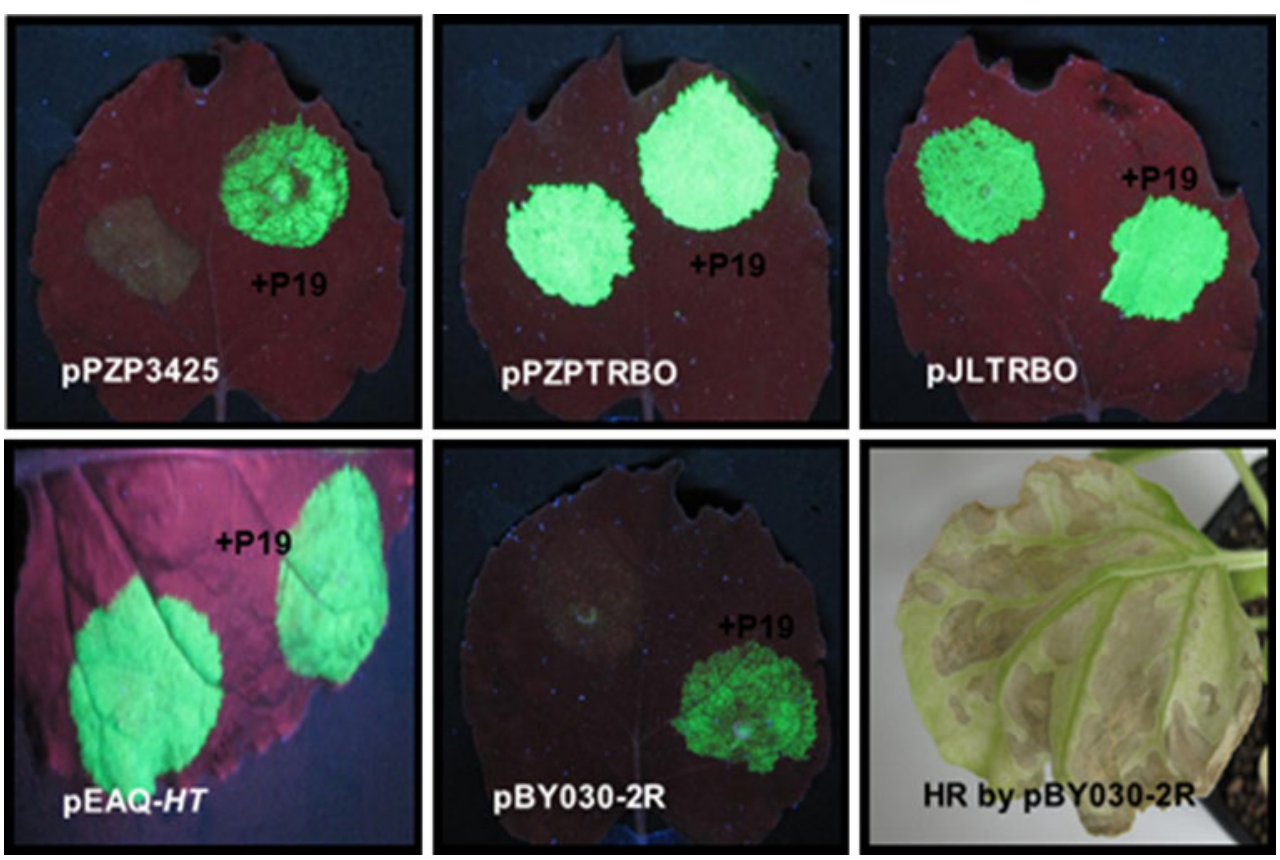


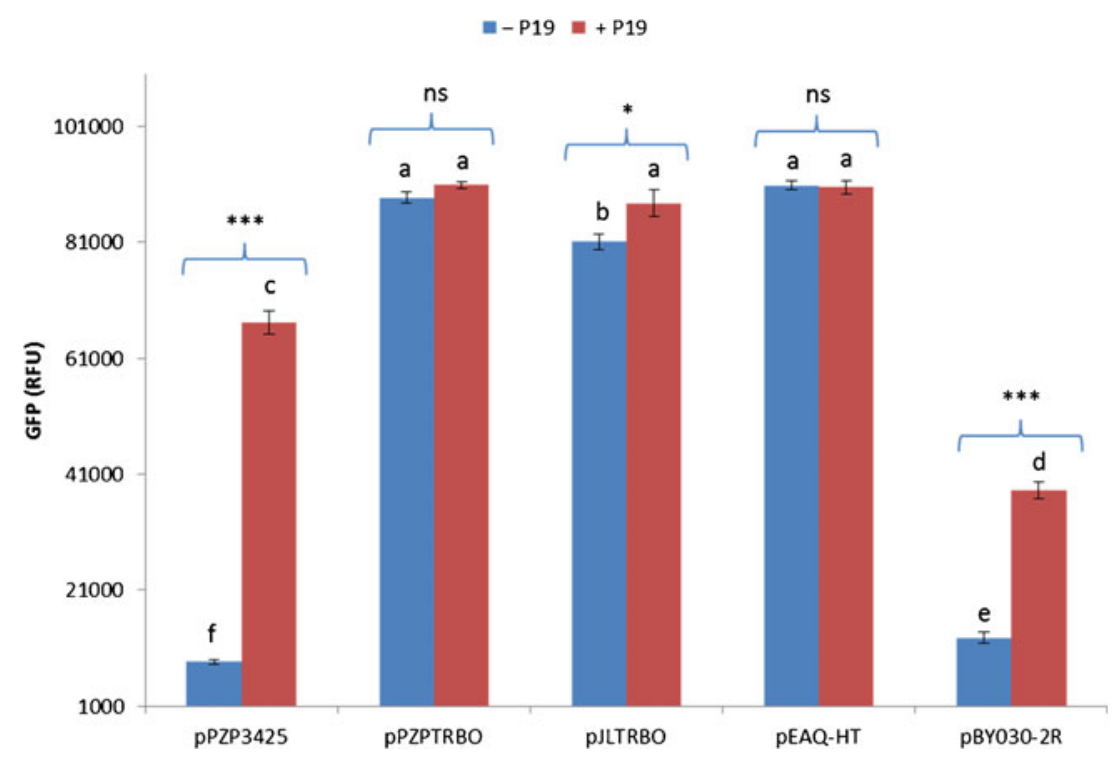

Fig. 3 Relative GFP fluorescence in N. benthamiana leaves infiltrated with different vectors. GFP relative fluorescence for different vectors with and without $\mathrm{P} 19$. The $\mathrm{OD}_{600}$ of the agrobacteria suspension used in this experiment was 1.0. The samples were taken at $5 \mathrm{dpi}$ to extract TSP for fluorescence measurement. The values $(R F U$ relative fluorescence unit) shown here are average \pm standard error (SE) of three individual

strongest effect when P19 was co-infiltrated, resulting in an expression level of about $75 \%$ of that of the TMV vectors and of pEAQ- $H T$. Infiltrating the agrobacteria at an $\mathrm{OD}_{600}$ of 0.4 resulted in a much lower GFP expression level for pPZP3425, while the TRBO vectors reached a level comparable to infiltration with $\mathrm{OD}_{600}$ of 1.0 , with the difference that the co-infiltration of P19 significantly enhanced the expression level (Fig. S5). The other experiments were therefore done with an Agrobacterium suspension of $\mathrm{OD}_{600}$ of 1.0. GFP measurements were confirmed by extracting total soluble protein from infiltrated leaves and resolving the proteins by SDS-polyacrylamide gel electrophoresis (PAGE) (Fig. S6).

Next, expression of the different vectors was compared using the GUS marker. Since pPZP3425 performed better than expected in GFP expression, an additional pPZP binary vector, pPZP5025, was included. Figure 4(a) shows representative pictures of the GUS-containing vectors after staining the leaves with X-Gluc. The colour figures did not show strong differences in staining for all vectors except pBY030$2 \mathrm{R}$ which gave a lower GUS expression level. The difference in GUS expression was easier to observe in black and white (Fig. 4(b)). The expression level of the binary pPZP vectors without P19 was much lower as compared to co-infiltration with P19. The virus vectors except pBY030-2R all had a strong expression level with and without P19. Quantifying the GUS expression by measuring GUS activity (Fig. 5) confirmed the GUS staining. The vector pBY030-2R had the lowest expression level, and co-infiltration with P19 doubled the GUS expression. Both TMV vectors together replicates. The data were analysed by one-way ANOVA $(p<0.05)$ and least significant difference (LSD) test using the Statistical Package for the Social Sciences (SPSS) software where $n=6$. The stars show a significant difference between samples with and without RNA-silencing inhibitor P19; different letters indicate a statistically significant difference, while $n s$ means no significant difference

with $\mathrm{pEAQ}-H T$ showed the strongest GUS expression. There was no difference in expression levels with or without P19 for $\mathrm{pPZPTRBO}$ and $\mathrm{pEAQ}-H T$, while $\mathrm{pJLTRBO}$ showed a significant increase in GUS activity with P19. Both pPZP binary vectors gave low-level GUS expression without P19, while co-infiltration of P19 resulted in a GUS expression level that was comparable to that of the TMV vectors and the pEAQ-HT vector. TSP samples extracted from GUSexpressing leaves were also resolved on SDS-PAGE showing a good correlation between the levels of GUS activity in the assays and GUS protein present in the SDS-PAGE gels (Fig. S7).

\section{Discussion}

Transient expression in plants has become a widely used technique in basic research and for the production of proteins to be used in applied research. Since the first report of Agrobacterium-mediated transient expression of a GUS binary vector in bean leaves (Kapila et al. 1997), interest has shifted to viral vectors which are delivered by agroinfiltration and which can replicate in the infiltrated plants. Here, we have compared the efficiency of several viral vectors together with simple binary vectors. The magnifection vectors (Gleba et al. 2005, 2007; Marillonnet et al. 2005) were excluded because these are not freely available. This TMV system consists of different pro-vectors which are delivered by agrobacteria to the plant cell where they recombine to produce the final expression vector. 

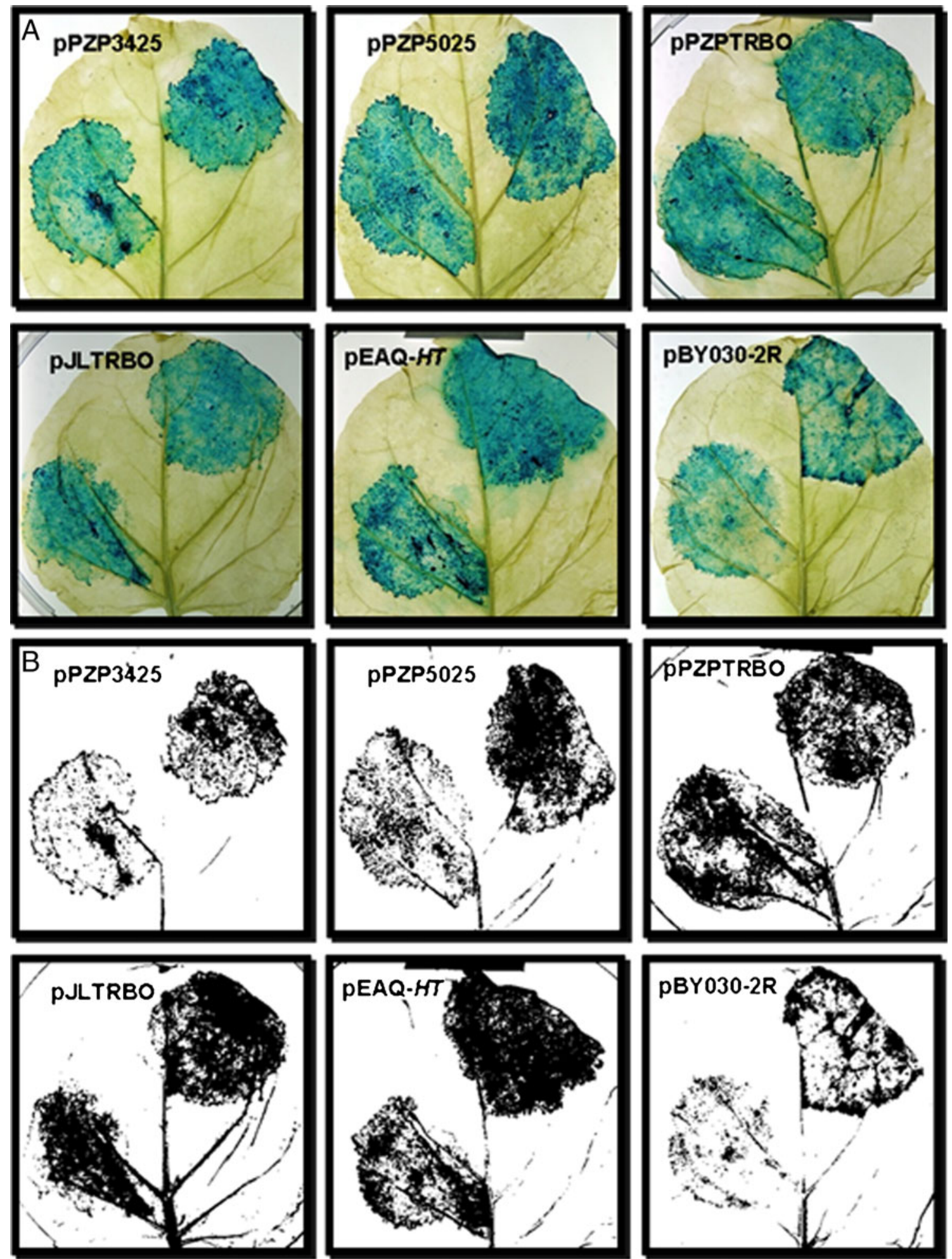

Fig. 4 GUS expression in $N$. benthamiana leaves infiltrated with different vectors. $A$ GUS staining was performed at 5 dpi. The $\mathrm{OD}_{600}$ of the agrobacteria suspension used for infiltration was 1.0. The right

As expected, the binary vectors pPZP3425 and pPZP5025 performed poorly without the co-infiltration of an RNAi inhibitor (Fig. 5). For GFP, expression without P19 reached side of each leaf in the image was co-infiltrated with $\mathrm{P} 19$, while the left side is without $\mathrm{P} 19 ; B$ black and white image of $A$ to make the difference of GUS expression levels clear

only $10 \%$ of the expression level of pPZP3425 with P19, while for GUS, both reached $30 \%$ without $\mathrm{P} 19$ as compared to expression with P19. It has been shown before that RNAi 


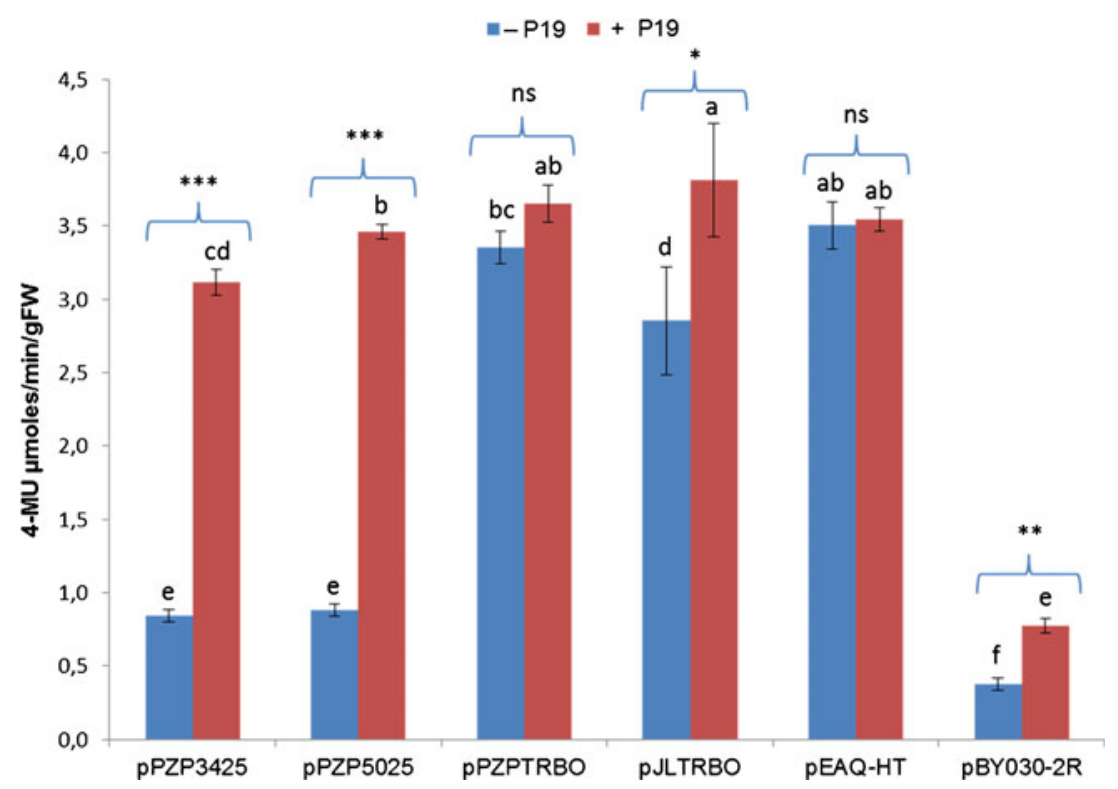

Fig. 5 GUS activity assay for different expression vectors. The GUS activity measurement with different vectors infiltrated with or without RNA-silencing inhibitor P19 was done according to standard protocols. The $\mathrm{OD}_{600}$ of the agrobacteria suspension used in this experiment was 1.0. The samples were taken at $5 \mathrm{dpi}$ to extract TSP and were used for GUS activity assays. The values shown are average $\pm \mathrm{SE}$ of the three

leads to a downregulation of protein expression in transiently transformed plant leaves after a few days and that this can be suppressed by co-infiltration of RNAi inhibitors (Voinnet et al. 2003). Lombardi et al. (2009) compared the three different RNAi inhibitors (P25 of potato virus X, P19 of either artichoke mottled crinkle virus or tomato bushy stunt virus) for their effect on transient expression of the Nef protein and found that the P19 protein of artichoke mottled crinkle virus gave the highest expression level. Here, the P19 inhibitor from pBIN61-P19 (Voinnet et al. 2003) was used which is also included in the pEAQ- $H T$ vector T-DNA (Sainsbury et al. 2009).

In contrast to the binary vectors, quantification of GUS and GFP expression (Figs. 3 and 5) showed that TRBO and the pEAQ- $H T$ vector gave similar results with and without co-infiltration of P19. pEAQ- $H T$ has an expression cassette for P19 integrated within the T-DNA, while TRBO does not contain any RNAi inhibitor. It has been reported before that TRBO expression does not need an RNAi inhibitor, and it was speculated that this was due to the deletion of the TMV coat protein open reading frame (ORF) and subgenomic promoter (Lindbo 2007). However, there was a small but significant effect of P19 co-infiltration on both GFP and GUS expressions in the case of TRBO. In the case of pPZPTRBO, this effect was only seen with GUS expression, not with GFP. The reason for this difference might be related to the higher copy number of pPZPTRBO. P19 had no effect on pEAQ- $H T$ expression in GV3101 but did result in an increased expression in the LBA4404 strain. individual replicates. The data were analysed by one-way ANOVA $(p<0.05)$ and LSD test using the SPSS software where $n=6$. The stars show a significant difference between samples with and without P19; different letters indicate a statistically significant difference, while $n s$ means no significant difference

The pBY030-2R vector gave much lower expression levels, both with GFP and GUS, even with co-infiltration of the P19 inhibitor as compared to TRBO and pEAQ-HT. The expression level was approximately $45 \%$ for GFP and $20 \%$ for GUS if used with P19 and approximately 15 and $10 \%$, respectively, without $\mathrm{P} 19$. It also performed less well than the pPZP vectors co-infiltrated with P19 (Figs. 3 and 5). An explanation why this vector gave the lowest expression levels in our experiments might be that it was designed to be used for the production of virion particles for vaccination (Huang et al. 2009). Since it contains the coat protein, this might be reducing the efficiency in the transient expression of other proteins.

The binary vectors pPZP3425 and pPZP5025 were included for comparison with the viral vectors and $\mathrm{pEAQ}-H T$. However, it turned out that their performance in our comparison was similar to that of TRBO and pEAQ- $H T$ if used together with P19. With the GFP marker, pPZP3425 gave $75 \%$ of the TRBO and pEAQ-HT expression level (Fig. 3), while the GUS protein was expressed at comparable levels by pPZP3425 and pPZP5025 (Fig. 5). This is especially interesting, because these vectors could also be used for stable transformation of plants. Thus, different constructs could, for instance, be tested by transient expression in $N$. benthamiana, and the best ones could then directly be used for stable transformation. This would also be possible with the pEAQ- $H T$ vector, but in that case, the P19 cassette which is included in the T-DNA would also be integrated into the plant genome, leading to a constitutive expression of P19 in the transgenic plants which is certainly not intended. 


\section{Conclusion}

The TMV RNA-based vector pJLTRBO and its derivative pPZPTRBO show good potential to produce recombinant proteins in plants without using the RNA-silencing inhibitor P19. The advantage of pPZPTRBO is that it is a high-copynumber plasmid, also in $E$. coli, making cloning easier. Similar expression levels were provided by the pEAQ- $H T$ vector which has an integrated P19 expression cassette. Binary pPZP vectors gave an expression level which was only $10-20 \%$ lower than TRBO or pEAQ- $H T$ and have the advantage that they can also be directly used for the production of transgenic plants.

Acknowledgments The authors thank Sabine Daxböck-Horvath and Martina Niese for their technical assistance; Dr. Shahid Masood Siddique, Amjad Abbas, Muhammad Amjad Ali and Stephan Plattner for their assistance in lab work; and Dr. Mason, Dr. Lindbo, Dr. Lomonossoff and Dr. Baulcombe for providing the vectors used in this study. This research was supported by a grant P21896-B16 from the Austrian Science Fund (FWF). Kausar Hussain Shah and Bachar Almaghrabi were supported by Higher Education Commission (HEC) of Pakistan and OEAD, respectively.

Open Access This article is distributed under the terms of the Creative Commons Attribution License which permits any use, distribution, and reproduction in any medium, provided the original author(s) and the source are credited.

\section{References}

Ali MA, Shah KH, Bohlmann H (2012) pMAA-Red: a new pPZPderived vector for fast visual screening of transgenic Arabidopsis lines at the seed stage. BMC Biotechnol 12:37

Castilho A, Bohorova N, Grass J, Bohorov O, Zeitlin L, Whaley K, Altmann F, Steinkellner H (2011) Rapid high yield production of different glycoforms of Ebola virus monoclonal antibody. PLoS One 6:e26040

Gils M, Kandzia R, Marillonnet S, Klimyuk V, Gleba Y (2005) Highyield production of authentic human growth hormone using a plant virus-based expression system. Plant Biotechnol J 3:613-620

Giritch A, Marillonnet S, Engler C, van Eldik G, Botterman J, Klimyuk V, Gleba Y (2006) Rapid high-yield expression of full-size IgG antibodies in plants coinfected with noncompeting viral vectors. Proc Natl Acad Sci USA 103:14701-14706

Gleba Y, Klimyuk V, Marillonnet S (2005) Magnifection-a new platform for expressing recombinant vaccines in plants. Vaccine 23:2042-2048

Gleba Y, Klimyuk V, Marillonnet S (2007) Viral vectors for the expression of proteins in plants. Curr Opin Biotech 18:134-141

Goodin MM, Zaitlin D, Naidu RA, Lommel SA (2008) Nicotiana benthamiana: its history and future as a model for plant-pathogen interactions. Mol Plant Microbe In 21:1015-1026

Hashimoto M, Komatsu K, Maejima K, Okano Y, Shiraishi T, Ishikawa K, Takinami Y, Yamaji Y, Namba S (2012) I Identification of three MAPKKKs forming a linear signaling pathway leading to programmed cell death in Nicotiana benthamiana. BMC Plant Biol 12:103
Hajdukiewicz P, Svab Z, Maliga P (1994) The small, versatile pPZP family of Agrobacterium binary vectors for plant transformation. Plant Mol Biol 25:989-994

Hoekema A, Hirsch PR, Hooykaas PJJ, Schilperoort RA (1983) A binary plant vector strategy based on separation of Vir-region and T-region of the Agrobacterium tumefaciens Ti-plasmid. Nature 303:179-180

Holsters M, Dewaele D, Depicker A, Messens E, Vanmontagu M, Schell J (1978) Transfection and transformation of Agrobacterium tumefaciens. Mol Gen Genet 163:181-187

Huang Z, Chen Q, Hjelm B, Arntzen C, Mason H (2009) A DNA replicon system for rapid high-level production of virus-like particles in plants. Biotechnol Bioeng 103(4):706-714

Jefferson RA, Kavanagh TA, Bevan MW (1987) Gus fusions-betaglucuronidase as a sensitive and versatile gene fusion marker in higher plants. EMBO J 6:3901-3907

Kanagarajan S, Tolf C, Lundgren A, Waldenström J, Brodelius PE (2012) Transient expression of hemagglutinin antigen from low pathogenic avian influenza A (H7N7) in Nicotiana benthamiana. PLoS One 7:e33010

Kapila J, DeRycke R, Van Montagu M, Angenon G (1997) An Agrobacterium-mediated transient gene expression system for intact leaves. Plant Science 122:101

Koncz C, Schell J (1986) The promoter of $\mathrm{T}_{\mathrm{L}}$-DNA gene 5 controls the tissue-specific expression of chimaeric genes carried by a novel type of Agrobacterium binary vector. Mol Gen Genet 204:383396

Lico C, Chen Q, Santi L (2008) Viral vectors for production of recombinant proteins in plants. J Cell Physiol 216:366-377

Lindbo JA (2007) TRBO: a high-efficiency tobacco mosaic virus RNAbased overexpression vector. Plant Physiol 145:1232-1240

Liu L, van Tonder T, Pietersen G, Davies JW, Stanley J (1997) Molecular characterization of a subgroup I geminivirus from a legume in South Africa. J Gen Virol 78:2113-2117

Lombardi R, Circelli P, Villani ME, Buriani G, Nardi L, Coppola V, Bianco L, Benvenuto E, Donini M, Marusic C (2009) High-level HIV-1 Nef transient expression in Nicotiana benthamiana using the P19 gene silencing suppressor protein of artichoke mottled crinckle virus. BMC Biotechnol 9:96

Marillonnet S, Thoeringer C, Kandzia R, Klimyuk V, Gleba Y (2005) Systemic Agrobacterium tumefaciens-mediated transfection of viral replicons for efficient transient expression in plants. Nat Biotechnol 23:718-723

Mor TS, Moon YS, Palmer KE, Mason HS (2003) Geminivirus vectors for high-level expression of foreign proteins in plant cells. Biotechnol Bioeng 81:430-437

Pogue GP, Vojdani F, Palmer KE, Hiatt E, Hume S, Phelps J, Long L, Bohorova N, Kim D, Pauly M, Velasco J, Whaley K, Zeitlin L, Garger SJ, White E, Bai Y, Haydon H, Bratcher B (2010) Production of pharmaceutical-grade recombinant aprotinin and a monoclonal antibody product using plant-based transient expression systems. Plant Biotechnol J 8:638-654

Popescu SC, Popescu GV, Bachan S, Zhang ZM, Seay M, Gerstein M, Snyder M, Dinesh-Kumar SP (2007) Differential binding of calmodulin-related proteins to their targets revealed through high-density Arabidopsis protein microarrays. Proc Natl Acad Sci USA 104:4730-4735

Rymerson RT, Menassa RA, Brandle JE (2002) Tobacco, a platform for the production of recombinant proteins. In: Erickson $\mathrm{L}, \mathrm{Yu}$ W-J, Brandle J, Rymerson R (eds) Molecular farming of plants and animals for human and veterinary medicine. Kluwer, Amsterdam, pp 1-31

Sainsbury F, Lavoie P, D’Aoust M, Vezina L, Lomonossoff G (2008) Expression of multiple proteins using full-length and deleted versions of cowpea mosaic virus RNA-2. Plant Biotech J 9:8292 
Sainsbury F, Thuenemann E, Lomonossoff G (2009) pEAQ: versatile expression vectors for easy and quick transient expression of heterologous proteins in plants. Plant Biotechnol J 7(7):682-693

Schägger H (2006) Tricine-SDS-PAGE. Nat Protoc 1:16-22

Sheludko YV, Sindarovska YR, Gerasymenko IM, Bannikova MA, Kuchuk NV (2007) Comparison of several Nicotiana species as hosts for high-scale Agrobacterium-mediated transient expression. Biotech Bioeng 96:608-614

Scholthof K-BG (2004) Tobacco mosaic virus: a model system for plant biology. Annu Rev Phytopathol 42:13-34

Szakasits D, Siddique S, Bohlmann H (2007) An improved pPZP vector for Agrobacterium-mediated plant transformation. Plant Mol Biol Rep 25(3-4):115-120

Twyman RM, Stoger E, Schillberg S, Christou P, Fischer R (2003) Molecular farming in plants: host systems and expression technology. Trends Biotechnol 21:570-578
Van der Hoorn R, Laurent F, Roth R, De Wit P (2000) Agroinfiltration is a versatile tool that facilitates comparative analyses of Avr9/Cf-9induced and Avr4/Cf-4-induced necrosis. Mol Plant Microbe In 13:439-446

Vaquero C, Sack M, Chandler J, Drossard J, Schuster F, Monecke M, Schillberg S, Fischer R (1999) Transient expression of a tumorspecific single-chain fragment and a chimeric antibody in tobacco leaves. Proc Natl Acad Sci USA 96:11128-11133

Voinnet O, Rivas S, Mestre P, Baulcombe D (2003) An enhanced transient expression system in plants based on suppression of gene silencing by the p19 protein of tomato bushy stunt virus. Plant J 33:949-956

Yang S, Carter S, Cole A, Cheng N, Nelson R (2004) A natural variant of a host RNA-dependent RNA polymerase is associated with increased susceptibility to viruses by Nicotiana benthamiana. Proc Natl Acad Sci USA 101:6297-6302 